\title{
CERÁMICA AZUL Y DE REFLEJO DORADO EN ELCHE: CONJUNTO DE SAN JOSÉ
}

\author{
Grupo llicitano de Estudios Arqueológicos
}

El Grupo llicitano de Estudios Arqueológicos está compuesto por los siguientes miembros:
M. ${ }^{a}$ Carmen Albaladejo Pomares
Pascual Bolaños Argilés
Josefa Caballero Segarra
Fermín Crespo Rodríguez
Marga Durá i Guilló
Araceli Hoffman Javaloyes
Juan Antonio León Fabrellas
Marga Martínez García
Rafael Martínez García
Raquel Pastor Clement
Pedro Javier Ripoll Vivancos
Inmaculada Ruiz Pascual
José Antonio Sáez Zaragoza
Julián Salazar Vives
Juan Jesús Vallejo Padilla

\section{APARICIÓN DE LOS MATERIALES}

Los materiales que se analizan en este estudio aparecieron en una pequeña loma situada entre el Convento de San José y el cauce del río Vinalopó, no estando separada en la actualidad por más de una quincena de metros del primero. Presenta en sus lados Norte y Este sendos terraplenes, generando un fuerte desnivel que intentó ser contenido por un murete que la circundaba por tres de sus lados; mostrándose en el cuarto de ellos y más próximo al convento, un corte vertical producido, en este caso, por las obras de nivelación del terreno llevadas a cabo con motivo de la construcción del actual complejo cultural. 


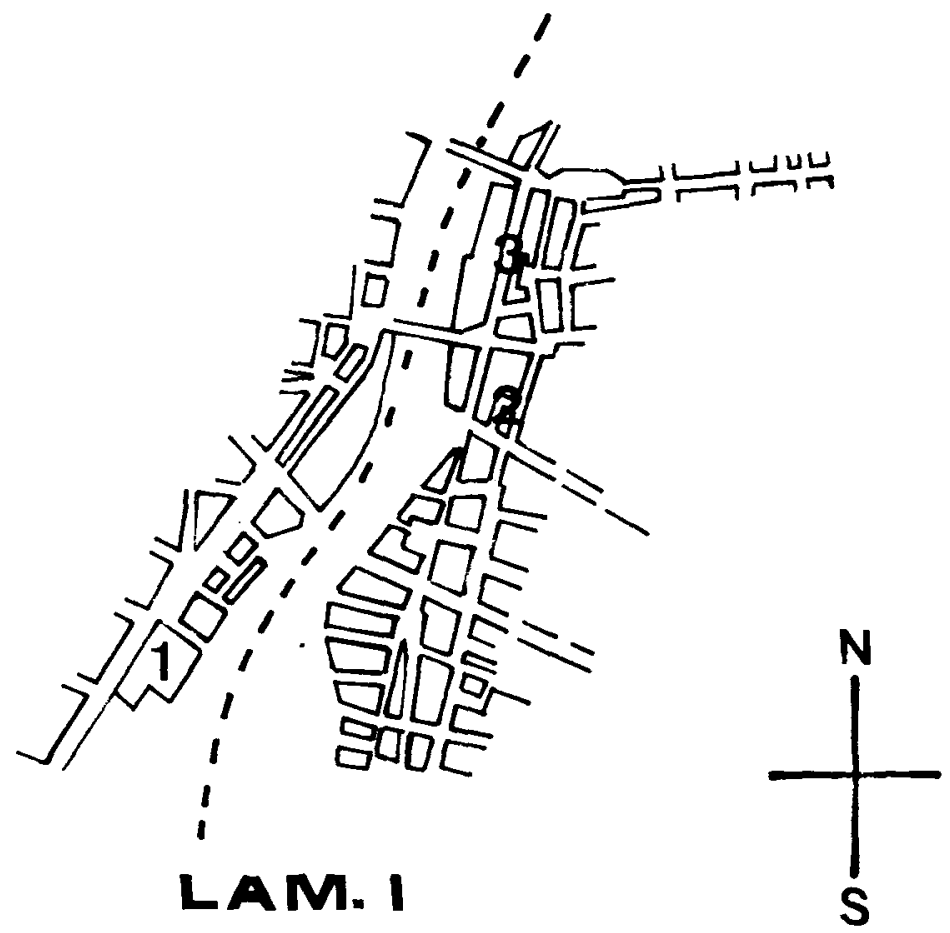

Lámina I. 1.-Convento de San José. 2.-Pobla de Sant Jordi. 3.-Huerto de Gil.

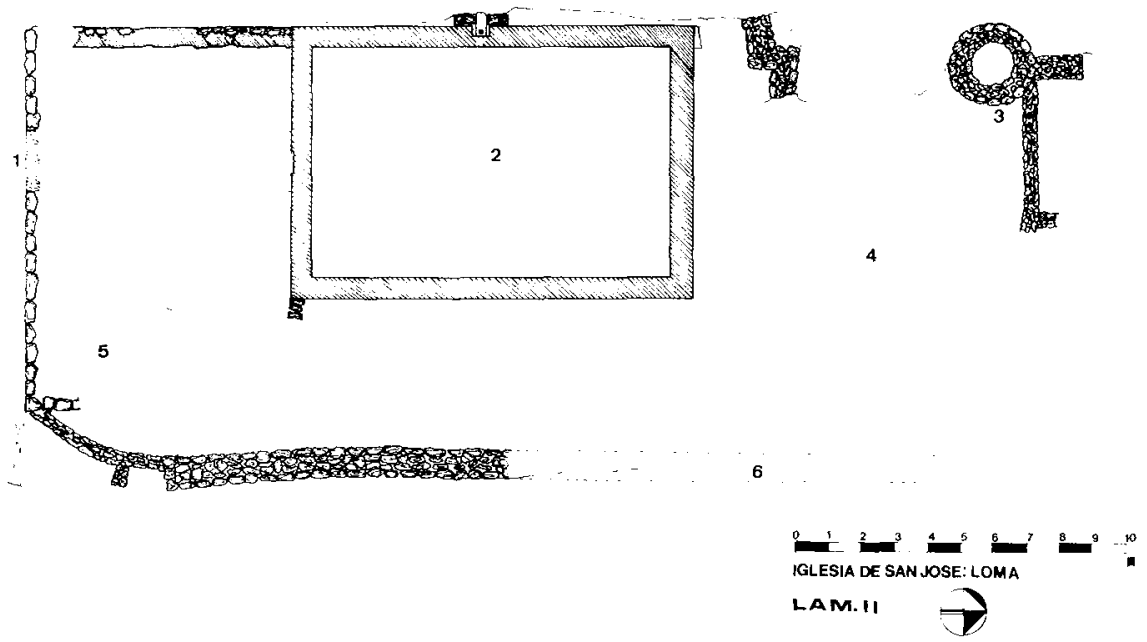

Lámina II. Planta de la loma. 1.-Construcción abovedada. 2.-Balsa. 3.-Pozo y restos de muretes. 4 y 5.-Zonas de concentración de humedad. 6.-Muro de contención desaparecido en parte. 
La loma en cuestión es de planta rectangular, su máxima longitud (dirección N-S) es de unos $35 \mathrm{~m}$. aproximadamente, en cuanto a su lado transversal (dirección E-W) tiene unos $15 \mathrm{~m}$., con una altura de cerca de 4,5 m. por su cara Este.

En la superficie encuadrada por dicha loma encontramos una balsa rectangular de $13,6 \mathrm{~m}$. de larga por $8,5 \mathrm{~m}$. de ancha, cuyo lado mayor está situado en el borde Oeste de la misma, efectuada en mortero de cal y cuyo alzado sólo se conserva hasta ras de suelo, y en la actualidad colmatada hasta éste.

También nos encontramos un recinto abovedado que, aprovechando el desnivel existente en la parte Sur, se adentra bajo la loma. Presenta una longitud de $8 \mathrm{~m}$. por 1,5 de anchura y 1,5 m. de altura, estando construido en mampostería. En él tanto el suelo como la bóveda están recubiertos de ladrillos macizos, encontrándose en el lienzo frontal del recinto una pequeña hornacina de fondo plano.

Durante las obras de restauración y ampliación del Convento franciscano de San José para su utilización como complejo cultural, y más concretamente el 20 de enero de 1979, comienza a llamar la atención la aparición de restos de unas construcciones en la loma situada al Este de aquél.

Posteriormente, en septiembre de 1983 se hallan en una de dichas construcciones restos de ladrillos macizos, lo que llevó a algunos vecinos de la zona a comunicar a la prensa (1) el descubrimiento de un posible horno. El 10 de septiembre, este grupo amplió la noticia en el Diario Información, descartando la posibilidad de que se tratase de un horno al no encontrarse restos de ladrillos refractarios.

A pocos metros de la mencionada construcción, el día 12 del mismo mes, comenzaron a aparecer en el lado SW de la loma los restos de loza que aquí estamos tratando, a consecuencia de un pequeño desprendimiento de un lateral de la misma provocado por las lluvias.

\section{RESEÑA HISTÓRICA DEL CONVENTO DE SAN JOSÉ}

Según nos cuenta Pedro Ibarra i Ruiz, «el día 2 de febrero de 1561 y en el Capítulo congregado por los penitentes hijos descalzos del Serafín llagado San Francisco en el Convento del Pedroso, el glorioso Doctor y Padre San Pedro de Alcántara, eligió ocho religiosos para que vinieran a Elche a fundar un convento de su Orden» (2).

Fueron recibidos por el Justicia y Regimiento de la Villa y por el pueblo congregado para su recepción, siendo acompañados, tras la visita a Santa 
María, al lugar donde se edificaria el convento y que por aquel tiempo ocupaba una ermita dedicada a San José.

No queda en la actualidad ningún resto de aquel primitivo edificio fundado por el Marqués de Elche; «los sucesores del Marquesado fueron devotísimos patronos del Convento, cuyos frailes se dedicaron singularmente a la curación de enfermos y a la Enseñanza» (3).

«Fue este Convento Cabeza de la Provincial hasta el 4 de mayo de 1574, en cuya fecha se fundó el Convento de San Juan de Ribera, en Valencia, que por ser la capital adquirió este título» (4).

Dado que el convento se hallaba situado en la margen derecha de la rambla del Vinalopó y que ésta significaba una importante separación del resto de la población, que en las épocas de avenidas, heladas, etc, suponían el aislamiento de los frailes, puesto que la construcción del puente de Santa Teresa no comenzó hasta 1705, era deseo de la comunidad franciscana y también de la Villa, "establecer su morada en sitio más en contacto con la población» (5). Así, en 1613, se instó, con la autorización eclesiástica, el traslado de la comunidad. Sin embargo, esto no habría de tener lugar hasta que durante la trágica peste de 1647-48, el convento se vio afectado también por la epidemia. Por consejo de la Junta Local de Sanidad, el Ayuntamiento decidió desalojar las reducidas e infectas salas del Hospital Municipal «y trasladar a los apestados a las espaciosas y bien ventiladas habitaciones del Convento de S. José» (6), y así mismo, alojar a la comunidad en la Casa de los Malla, existente al fondo del callizo de la Alpujarra, lo que tuvo lugar el 23 de junio de 1648 (7).

Por parte del Ayuntamiento «se dio conocimiento del suceso al señor Duque, Marqués de Elche, Patrono de la institución, reseñándose la perentoria necesidad que les obligó al traslado, lo que estaban prontos a solucionar edificando el Ayuntamiento otro convento en el sitio que mejores condiciones reuniera a beneplácito de todos, pudiendo continuar desde luego, S.E. con el honor del Patronato, sin obligación de acudir para nada en la construcción de la nueva obras (8).

Pero el Duque ni aprobó el traslado ni quiso oír las razones expuestas por la corporación municipal, por lo que el asunto pasó a los Tribunales de Justicia. Al Ayuntamiento, no conveniéndole la disparidad de opiniones con aquél, hizo que los frailes volvieran a su convento, habiendo sido previamente restaurado y acomodado nuevamente a la vida monástica. En este sentido, la Sitiada de 18 de noviembre de 1650 , hace referencia a que tras la utili- 
zación del convento como hospital para el tratamiento de los contagiados, se ordena la quema de la ropa de éstos para purificar el lugar y que pueda ser ocupado de nuevo por los frailes (9).

«Del año 1650 es la escritura de Patronato del expresado convento de San José por los Duques, Marqueses de Elche» (10), que eran la base de financiación del mismo (11). El actual edificio es de 1678, siendo el anterior completamente derruido, habiendo con posterioridad una petición de la Orden Tercera para recabar fondos a fin de construir otra nave (12).

La comunidad, que en algún momento llegó a contar con 54 religiosos, se dedicaba a distintas artes y oficios, destacando la enseñanza superior.

Según el Cabildo de 20 de agosto de 1835 se procede al «cierre de los conventos y orden de salida de estos religiososm. No obstante, Ramos Folqués indica que la supresión de los conventos se decreta el 8 de marzo de 1836 y como consecuencia son expulsados los frailes, pidiendo el Ayuntamiento en 1837, la concesión del Convento de S. José como Casa Hospital de Beneficiencia (13), petición que se volvió a formular en 1841 ante las malas condiciones del hospital de la Corredora (14), siendo conseguida la cesión según Real Orden de 11 de junio de 1841, tomando el Ayuntamiento posesión inmediata del edificio.

\section{APROXIMACIÓN A LA ALFARERÍA EN ELCHE}

Dado que el objeto del presente estudio es dar a conocer un pequeño conjunto de materiales cerámicos encuadrados en los siglos XVII y XVIII, es de todo punto necesario el hacer siquiera un breve análisis de la industria alfarera en la ciudad de Elche, para lo cual habremos de remontarnos a 1438, fecha en que tenemos la primera referencia de la llegada a la villa de un "Mestre de fer teula e rajola" (15). A partir de dicha referencia podemos constatar documentalmente una industria alfarera que perdura durante los siglos $X V$ al $X \mid X$, con una clara interrupción en el XVIII.

No obstante, la escasez cuantitativa de las noticias y su parquedad para un espacio temporal tan amplio, así como el incipiente estado de las investigaciones en esta materia, dificultan por ahora elaborar un análisis de fondo sobre el tema. Sin embargo, sí podemos intentar esbozar aqui algunos rasgos genéricos sobre este tipo de industria, a pesar de no haber sido ésta una de las más características dentro de las producciones artesanales de la villa.

Hemos de tener presente, ante todo, la excelente situación que presenta la villa de Elche, al estar asentada en el cono de deyección del río Vinalopó, 
lo cual implica la presencia de arcillas, materia prima para esta industria, como bien se refleja en la petición de asentamiento de un maestro alfarero en 1462 (16).

La ubicación de la industria cerámica dentro de la estructura urbana va a estar claramente determinada por su carácter polucionante, por ello los alfares se construirán algo alejados de la "vila murada», núcleo urbano principal, como el resto de industrias con análogo carácter (curtidores, jabonerías, tintorerías, hornos de vidrio (17), ...), concentrándose aguas abajo de la ciudad, dentro del anillo periurbano de la misma. Por la necesidad de agua para la fabricación, y por las impurezas que aquélla adquiere tras su uso, los talleres de ceramistas se van a situar cerca de las acequias, una vez concluido el trayecto urbano de las mismas. Este tipo de asentamiento de las industrias polucionantes era ya habitual en el esquema urbanístico musulmán (18). En Elche tenemos noticia de distintos asentamiento de esta industria, en el huerto de Gil (19), en la Plaça de Sant Jordi (20), en el Carrer de les Barques (21), es decir, en el barrio de la Pobla de Sant Jordi, asentamiento mudéjar hasta el XVI (22), y finalmente una última referencia de petición de asentamiento junto al desamortizado Convento de San José, en aquellos momentos Hospital de Caridad, próximo a la acequia de Marchena (23).

En cuanto a la importancia económica de esta industria en la ciudad, las referencias son escasas, aunque podemos, sin embargo, adivinar que se mantiene una evolución constante. Hubo de ser esta industria, cuando menos, atractiva ya que hay bastantes peticiones de asentamiento por parte de personas de dicho oficio y el Concejo las concede rápidamente, dando grandes facilidades a los peticionarios (24). Hay incluso alfareros que vienen temporalmente a trabajar a la ciudad y el Consejo paga su alojamiento (25). De estos dos hechos podríamos llegar a colegir que generalmente la demanda supera a la producción. Así mismo, podemos observar que desde el siglo XVII ya no se hace referencia a la construcción de hornos, lo que unido a la escasez de datos sobre el siglo XVIII podría indicar cierta decadencia de la industria alfarera, hasta el punto de que el Ayuntamiento llega a vender la canterería por mal estado en 1677 (26). Así pues, ya no tendremos noticias de petición de establecimiento de un alfarero hasta 1853 (27), habiendo de señalar en este punto que durante el siglo XVIII no aparece ninguna referencia a la industria alfarera ni el Padrón de 1761, ni en los Libros de Padrones y Cabreves de 1787, como tampoco en las Matrículas de 1835-50 (28), aunque sí hay noticias de un cierto comercio, si bien no podemos precisar si la producción es de origen local o no.

Por último, también puede dar una idea de la relativamente escasa importancia dentro de la industria local el dato de que en 1684 los alfareros soliciten acogerse a los estatutos de otro gremio como es el de los carpinteros (29). 


\section{LOS MATERIALES}

Hemos de hacer constar que al no tratarse de un conjunto perteneciente todo él a una misma unidad estratigráfica $o$, por lo menos, no contamos con ese dato, y más bien, en base tanto a la forma de aparición de la cerámica como a la existencia de diversos fragmentos pertenecientes al $\mathrm{XIX}$, tenemos bastante certeza de lo contrario. De aquí que debamos ser extremadamente cautos al realizar cualquier datación.

Así pues, la sistematización del estudio de la cerámica, ha sido tratada desde un punto de vista meramente tipológico. En primer lugar, haremos referencia a la cerámica de reflejo dorado, para pasar después a la cerámica decorada en azul sobre barniz blanco, obviando el resto de fragmentos aparecidos a causa de su escasa representatividad cuantitativa.

\section{A. LOZA DORADA}

En España, muestras de cerámica dorada aparecen a partir del siglo XI en Medina Azahara, desde el siglo XII en Calatayud y Málaga, y en Granada desde el S. XIV, concretamente conocemos que Pere Boill, señor de Manises, es enviado como embajador de Jaime ll ante la corte granadina en 1308-9 y que su hijo, Ramón Boïll, en 1335 comercia entre Valencia y Granada (30).

Desde el momento en que se lleva la técnica del reflejo dorado a Mani-, ses, será característico el reflejo nacarado de esta cerámica, que vive sus momentos de máximo esplendor durante los siglos XIV al XVI, siendo en este último siglo cuando se introduce el uso del molde.

Con la expulsión de los moriscos en 1609 degenera la técnica por falta de mano de obra. A partir de entonces la cerámica, más que dorada, se hace claramente cobriza (31).

En el año 1617 aparecen unas Ordenanzas por las que sólo se permite cocer obra de reflejo dorado a los maestros en dicho arte. Sin embargo, esta prohibición es reiterada por el gremio de los «escudellers» un siglo más tarde, lo que prueba que no habia sido cumplida y que probablemente circularian unas piezas doradas similares a las de Manises, pero más vulgares (32).

En cuanto a los motivos decorativos más típicos, en el S. XV son características las hojas de cardo "a la manera de Damasco", en el centro del plato; en el S.XVI aparecen cordoncillos y tetones junto a solfas, a manera de nota musical, que se van repitiendo. En el XVII no existe un repertorio muy amplio y sí un manifiesto shorror vacui» que cubre todo el anverso de los platos e, 
incluso, en ocasiones, el reverso, aunque aquí la decoración ya no es tan abigarrada; es frecuente el motivo de hojas, representadas de perfil, con bordes en sierra y terminación central en espiral, ocupando prácticamente toda la superficie. También son características de este siglo unas hojas, más pequeñas que las anteriores y utilizadas como motivo secundario, con el interior rayado, y que salen de un mismo tallo o de varios que se unen formando una trenza; otros motivos florales, también frecuentemente utilizados, eran el de la flor de Lis, los ramilletes de pequeños claveles y las hojas filamentosas. Muy frecuente hasta mediados de siglo era el motivo del "pardalot», gran pájaro con el cuerpo cubierto con líneas que quieren imitar el plumaje; aparece dibujado sobre fondo de tallos y hojas redondeadas. Otra figura animal también bastante utilizada era la del león. Sin embargo, en este siglo, como ya hemos señalado, empiezan a darse las primeras muestras de la decadencia de este tipo de cerámica, manifestada en la menor calidad del reflejo utilizado, que comienza a ser rojizo, así como la falta de imaginación en los dibujos. A mediados de siglo comienzan también los ensayos de policromía unido a un cambio de fondos, en los que aparecen grandes hojas alternando con otros motivos florales y una especie de frutas triangulares y, a veces, salpicado de puntos dorados. Las formas más usuales de este siglo son el plato de $35 \mathrm{~cm}$. de diámetro y el de $19 \mathrm{~cm}$., conocidos estos últimos como catavinos, pues se cree que eran colocados bajo el grifo de los barriles o bien servían de soporte a las medidas y recogían el vino que rebosaba de ellas. Sus paredes son relativamente gruesas, marcadas en la parte externa por las acanaladuras del torno y, en el centro del anverso, por la marca provocada por el «atilfe», artilugio que servía para la colocación separada de las piezas apiladas en el horno y que dejaba como huella tres puntos formando triángulo. Son asi mismo piezas muy pesadas en relación a su tamaño (33).

En el siglo XVIII con reflejo dorado tan sólo se harán unas pocas piezas, fundamentalmente catavinos, y otros platos grandes destinados a tapar la boca de las tinajas, se trata además de un reflejo cobrizo de muy baja calidad, con la típica y conocida decoración del "pardalot» y, rara vez, algún ciervo. Se desarrolla, por el contrario, todo tipo de loza policromada (34).

Así pues, podemos afirmar que las piezas cerámicas aparecidas en el Convento de San José y signadas como 01 y 02 podrían considerarse catavinos y fecharse como Manises de finales del siglo XVII. Apoyaría también esta afirmación la existencia en los fondos del Museo Nacional de Cerámica González Martí de Valencia de una pieza signada con el número 1.678, expuesta en la vitrina VI, de las mismas características.

Dentro de este apartado, pero al magen de los dos catavinos ya reseñados, hemos de hacer obligada referencia al conjunto de fragmentos constitui- 
do por las piezas signadas con los números: $54,55,56,57,58,59,60,61$, 62,63 . Estos materiales pueden ser datados en el $\mathrm{s}$. XVIII gracias al análisis comparativo tanto de decoración como de formas establecido entre ellos y los fondos del Museo Nacional de Cerámica «González Martís de Valencia. No obstante debemos efectuar cierta matización puesto que los fragmentos signados con los números: $57,59,62$, podrían pertenecer con bastante certeza a fechas anteriores, al presentar motivos decorativos no plenamente característicos del citado siglo.

\section{B. LOZA DE REFLEJO METÁLICO DORADO: DESCRIPCIÓN DE MATERIALES}

N. ${ }^{\circ}$ Sig. 001. Catavino conservado casi en su totalidad. Presenta el motivo del "Pardalot" de alas desplegadas, ojo redondo y desproporcionado, cuerpo rayado y plumones en la cola, sobre fondo de puntos y volutas. Circundado por tres filetes al borde, siendo el exterior de mayor grosor. Todo ello aplicado sobre esmalte blanco.

Dim. 18,8 Máx; $5 \varnothing$ Mín; $5 \mathrm{H}$. cm.

N. ${ }^{\circ}$ Sig. 002. Catavino conservado casi en su totalidad. Presenta el motivo del «Pardalot», aunque en un deteriorado estado, sobre fondo de puntos. Circundado por tres filetes al borde, siendo el exterior de mayor grosor. Todo ello aplicado sobre esmalte blanco.

Dim.: 18,7 $\varnothing$ Máx; $6 \varnothing$ Mín; 5 H. cm.

N. ${ }^{\circ}$ Sig. 054. Fragmento indeterminado. Presenta al interior decoración vegetal y punteado separada por un filete, al exterior vegetal separada por una banda en volutas; tonalidad dorada sanguínea. Todo ello aplicado sobre esmalte blanco.

Dim. Máx: $13 \times 10,5 \mathrm{~cm}$.

N. ${ }^{\circ}$ Sig. 055 . Fragmento borde, de sección aplanada. Presenta al interior decoración vegetal y filetes al borde, al exterior filetes; tonalidad amarillenta al interior y dorada sanguínea al exterior. Todo ello aplicado sobre esmalte blanco.

Dim.: $18,4 \varnothing$ Máx; 2,5 H. cm.

N. ${ }^{\circ}$ Sig. 056. Fragmento base plana. Presenta al interior decoración vegetal, sin decoración exterior; tonalidad dorada sanguínea. Todo ello aplicado sobre esmalte blanco.

Dim. Máx: $3,5 \times 5,5 \mathrm{~cm}$.

N. ${ }^{\circ}$ Sig. 057. Fragmento borde, de sección redondeada. Presenta al interior decoración vegetal y punteado circundado por un filete, al exterior filete al borde; tonalidad dorada ocre. Todo ello aplicado sobre esmalte blanco.

Dim.: $19,5 \varnothing$ Máx; $2,5 \mathrm{H}$. cm.

N. ${ }^{\circ}$ Sig. 058. Idem. Presenta al exterior decoración de filete al borde. Todo ello aplicado sobre esmalte blanco.

Dim.: Máx. $6 \times 2,4 ; 20 \varnothing$ Máx cm.

N. ${ }^{\circ}$ Sig. 059. Idem. Presenta al interior decoración vegetal y punteado, remarcado por una banda a cada lado, circundado por filete al borde, al exterior ondas entrecruzadas; tonalidad dorada sanguinea. Todo ello aplicado sobre esmalte blanco.

Dim.: 19,5 Ø Máx; 5,5 H. cm.

N. ${ }^{\circ}$ Sig. 060. Fragmento soporte u orejera. Presenta al interior decoración indeterminada; tonalidad marrón e indicios de azul. Todo ello aplicado sobre esmalte blanco.

Dim.: $5,5 \times 3,3 ; 1,5 \mathrm{H}$. cm. 

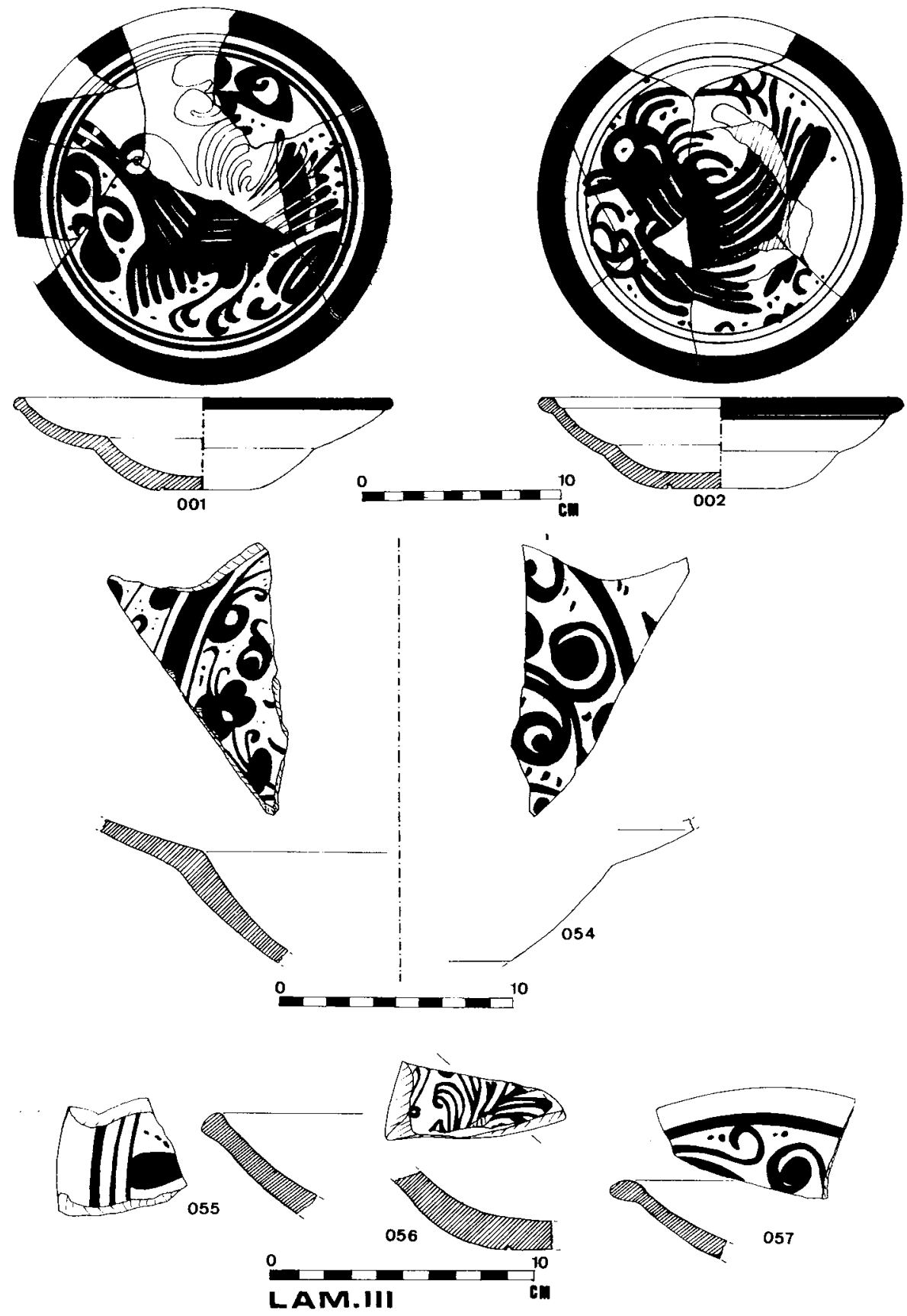

Lámina III. Loza dorada. 
N. ${ }^{\circ}$ Sig. 061 . Fragmento con borde y base, sección borde redondeada y base con anillo basal. Presenta al interior decoración vegetal y volutas, circundado por filetes al borde, al exterior vegetal y punteado, circundado por filete al borde y anillo basal; tonalidad marrón. Todo ello aplicado sobre esmalte blanco.

Dim.: $14,8 \varnothing$ Máx; $6,4 \varnothing$ Mín; $5 \mathrm{H}$. cm.

N. ${ }^{\circ}$ Sig. 062. Fragmento borde, de sección redondeada. Presenta al interior decoración en volutas, circundad por filete al borde, al exterior ondas entrecruzadas; tonalidad dorada sanguínea. Todo ello aplicado sobre esmalte blanco.

Dím. Máx.: $6,5 \times 5,4 ; 2,5 \mathrm{H}$. cm.

N. ${ }^{\circ}$ Sig. 063 . Fragmento base plana. Presenta al interior decoración indeterminada y punteado; tonalidad dorada sanguínea. Todo ello aplicado sobre esmalte blanco.

Dim. Máx.: $3,5 \times 3 ; 1 \mathrm{H} . \mathrm{cm}$.

\section{LOZA AZUL}

Pasamos ahora a examinar el conjunto con más representatividad dentro del presente estudio. Se trata de los platos decorados en azul, con tema vegetal simple (la palmeta) en el anverso y parte interior del borde -excepto el sig. 03 que tiene una $\mathrm{R}$ como tema central-, todo ello sobre vidriado blan$\mathrm{Co}$, existiendo, por otra parte, dos fragmentos de fuente que también incluimos en este apartado por presentar semejante decoración y pasta.

Dada la homogeneidad tipológica de este conjunto, utilizaremos, a la hora de describir cada una de las piezas, una división sistemática atendiendo a la superficie conservada en cada caso. De este modo distinguimos un grupo de piezas cuya superficie conservada es superior a un $60 \%$ como son las sig.: 03, 04, 05, 06, 07, 08, 09.

Del segundo grupo, es decir, el de aquellos cuya superficie conservada es inferior al $60 \%$, diferenciamos tres subgrupos. En primer lugar, aquellos fragmentos de plato que presentan borde y base, aunque sólo sea incipientemente, como los sig.: 11, 12 y 13 ; con las mismas características, pero perteneciente a una fuente, tenemos el sig. 10. El segundo subgrupo lo integran aquellos fragmentos de plato que sólo presentan borde, como son los sig.: $22,23,24,25,27,28,29,30,31,32,33,34,35,36,37,38,39,40,41,42$, $43,44,45$; de iguales características, pero perteneciente a un borde de fuente, tenemos el sig. 26. El último subgrupo lo componen aquellos fragmentos que presentan sólo base, como son los sig.: 14, 15, 16, 17, 18, 19.

Las circunstancias del hallazgo y las características de la cerámica, sumamente común, no nos permiten establecer una datación certera; no obstante, el parámetro temporal se sitúa entre mediados del XVII y finales del XVIII, en base a las características tipológicas de la cerámica, salvo sig.: 32,33 que quizás sean del XIX. Ello nos ha permitido plantearnos dos grandes hipótesis de trabajo como son, en primer lugar, la posibilidad de que dicho conjunto 
perteneciera en su totalidad o en parte a un alfar tradicional de las proximidades (Biar, Agost) o, por otro lado, que fuese de fabricación local. Para ello hemos utilizado el estudio bibliográfico, la encuesta directa y el trabajo de archivo.

No obstante hemos de acentuar las cautelas a la hora de intentar fijar la procedencia de los materiales puesto que podríamos estar tratando dos producciones distintas pero semejantes tipológicamente.

La posibilidad de que este conjunto cerámico pudiese pertenecer al alfar tradicional de Agost hemos podido descartarla tras haber mantenido varias conversaciones con dos de los ceramistas tradicionales más importantes de la localidad, como son Emili Boix, que en la actualidad realiza un tipo de cerámica artística a partir de métodos y técnicas tradicionales, y Pedro Mollá, cuya tradición familiar se remonta a diez generaciones de alfareros, trabajando siempre cerámica común. De estas conversaciones con dichos alfareros y de la contemplación «in situ» de las producciones y de los barros, podemos concluir que tradicionalmente en esta villa se ha producido un tipo de cerámica muy corriente, centrada, sobre todo, en botijos, orzas y lebrillos, siendo extraña la producción de vajilla. En segundo lugar, es de notar que no existen producciones vidriadas. Por último, el dato más importante es que, a pesar de la variedad de barros utilizados históricamente, la característica común a todos ellos es que tras su cocción el color de la pasta es un blanco muy puro y fino en textura, que en nada se asemeja a la que aquí estamos tratando.

En cuanto a que los materiales pertenezcan al alfar tradicional de Biar, hemos de decir, en primer lugar, que según afirmaciones del señor Maestre, único alfarero tradicional que queda en la población, la fabricación de loza fina es relativamente reciente, habiéndose producido tradicionalmente un tipo de cerámica común preferentemente cacharrería. Hay que reseñar en este punto que, a partir del primer tercio del siglo XIX comienza a haber una producción de cerámica vidriada, aunque de baja calidad, coincidiendo con el establecimiento en tales fechas de la familia de aquél, proviniente de Manises, que inició este tipo de producción. Así mismo, Madoz confirma en 1847 la existencia de loza fina en Biar. Por último, en cuanto a la pasta, es de destacar que ésta presenta un color rosáceo característico, que salvo escasas excepciones no está representado en el conjunto de materiales que estudiamos, pudiendo deberse éstas a la aleatoriedad de las mezclas de arcillas.

En definitiva, la hipótesis que parece tener mayores visos de certeza es la de la pertenencia de este conjunto de loza azul a la producción alfarera local, aunque la posibilidad de que el grupo cerámico perteneciente a la se- 
rie de loza azul fuese del alfar que se solicitó instalar en las inmediaciones del convento en 1853 y que desconocemos si se concedió, deberíamos descartarla en todo caso, puesto que las características del conjunto no corresponden a la de la cerámica que se produce en este área a mediados del siglo XIX.

\section{LOZA AZUL: DESCRIPCIÓN DE MATERIALES}

N. ${ }^{\circ}$ Sig. 003. Plato. Presenta al interior decoración vegetal al borde y representación de «R» en parte central del solero. Todo ello aplicado sobre esmalte blanco que deja entrever la pasta.

Dim.: $17 \varnothing$ Máx; $8 \varnothing \operatorname{Mín} ; 3,2$ H. cm.

N. ${ }^{\circ}$ Sig. 004. Idem. Presenta al interior decoración vegetal al borde y palmeta central. Todo ello aplicado sobre esmalte blanco.

Dim.: $17 \varnothing$ Máx; 7,5 $\varnothing$ Mín; 4 H. cm.

N. ${ }^{\circ}$ Sig. 005. Idem.

Dim.: 16,5 Ø Máx; $8 \varnothing$ Mín; 3,5 H. cm.

N. ${ }^{\circ}$ Sig. 006. Idem.

Dim.: 15,7 Ø Máx; 7,5 Ø Mín; 3,2 H. cm.

(La parte central del solero está completamente deteriorada).

N. ${ }^{\circ}$ Sig. 007. Idem.

Dim.: 17,5 $\varnothing$ Máx; 8,5 $\varnothing$ Mín; 3,5 H.cm.

N. ${ }^{\circ}$ Sig. 008. Idem.

Dim.: 16,3 $\varnothing$ Máx; $8 \varnothing$ Mín; 3,5 H. cm.

N. ${ }^{\circ}$ Sig. 009. Idem.

Dim.: 16,5 $\varnothing$ Máx; $8 \varnothing$ Mín; 3,7 H. cm.

N. ${ }^{\circ}$ Sig. 010. Fragmento de fuente con base plana y borde de sección redondeada. Presenta al interior filete al borde y motivo central indeterminado, al exterior salpicaduras. Todo ello aplicado sobre esmalte blanco.

Dim.: 18,6 $\varnothing$ Máx; $8 \varnothing$ Mín; 3,5 H. cm.

N. ${ }^{\circ}$ Sig. 011 . Fragmento plato base plana y borde. Presenta al interior motivo simple al borde y palmeta central. Todo ello aplicado sobre esmalte blanco.

Dim.: $8 \varnothing$ Min; $3,5 \mathrm{H}$. cm.

N. ${ }^{\circ}$ Sig. 012. Idem.

Dim.: $16 \varnothing$ Máx; 8,4 Ø Mín; 3,8 H. cm.

(Parte central indeterminada y esmalte deja entrever la pasta, adquiriendo una tonalidad rosácea)

N. ${ }^{\circ}$ Sig. 013. Fragmento plato base plana y borde. Presenta al interior motivo simple al brde. Todo ello aplicado sobre esmalte blanco.

Dim. Max.: $5 \times 5,5 ; 3,2 \mathrm{H}$. cm.

N. ${ }^{\circ}$ Sig. 014. Fragmento plato base plana. Presenta al interior posible palmeta central. Todo ello aplicado sobre esmalte blanco.

Dim. Máx.: $7 \times 6,5 ; 2,5 \mathrm{H}$. cm.

N. ${ }^{\circ}$ Sig. 015. Idem. Presenta restos de azul, sobre esmalte blanco.

Dim. Máx.: $8 \times 6 ; 2 \mathrm{H} . \mathrm{cm}$.

N. ${ }^{\circ}$ Sig. 016. Idem.

Dim. Máx.: $5 \times 4: 2 \mathrm{H} . \mathrm{cm}$.

(Sin ninguna decoración) 
N. ${ }^{\circ}$ Sig. 017. Idem.

Dim. Máx.: 4 ×5,5; $3 \mathrm{H} . \mathrm{cm}$.

(Sin ninguna decoración)

N. ${ }^{\circ}$ Sig. 018. Fragmento plato base plana. Presenta al interior posible palmeta central. Todo ello aplicado sobre esmalte blanco.

Dìm. Máx.: $7,5 \times 6,5 ; 2,6 \mathrm{H} . \mathrm{cm}$.

N. ${ }^{\circ}$ Sig. 019. Idem.

Dim. Máx.: $2,5 \times 2,4 ; 2 \mathrm{H} \mathrm{cm}$.

(Sin ninguna decoración)

N. ${ }^{\circ}$ Sig. 020. Fragmento plato base plana. Presenta al interior motivo vegetal cubriente. Todo ello aplicado sobre esmalte blanco.

Dim. Máx.: $8 \times 4,5 ; 2,5 \mathrm{H}$. cm.

N. ${ }^{\circ}$ Sig. 021. Fragmento borde de plato de sección redondeada. Presenta al interior motivo vegetal. Aplicado sobre esmalte blanco.

Dim. Máx.: 7,5×6; $3 \mathrm{H} ; 24 \varnothing$ Máx. cm.

N. ${ }^{\circ}$ Sig. 022. Fragmento borde de plato de sección ligeramente apuntada. Presenta al interior motivo simple. Aplicado sobre esmalte blanco.

Dim. Máx.: 4,5×4; $2 \mathrm{H} ; 15,5 \varnothing$ Máx. cm.

N. ${ }^{\circ}$ Sig. 023. Idem.

Dim. Máx.: $6 \times 4 ; 2 \mathrm{H} ; 15,5 \varnothing$ Máx. cm.

(Borde de sección redondeada)

N. ${ }^{\circ}$ Sig. 024. Idem.

Dim. Máx.: 7,5×5; 2 H; $19 \varnothing$ Máx.cm.

N. ${ }^{\circ}$ Sig. 025 . Idem.

Dim. Máx.: $5 \times 2,5 ; 1 \mathrm{H} ; 15,5 \varnothing$ Máx. cm.

(Borde de sección redondeada)

N. ${ }^{\circ}$ Sig. 026. Idem.

Dim. Máx: $3,8 \times 2 ; 1,2 H ; 16,4 \varnothing$ Máx. cm.

(Filete al interior del borde)

N. ${ }^{\circ}$ Sig. 027. Idem.

Dim. Máx.: 3,5×3; 1 H; 19,6 Ø Máx. cm.

N. ${ }^{\circ}$ Sig. 028 . Idem.

Dim. Máx: $3 \times 2,3 ; 1,5 \varnothing$ Máx. cm.

N. ${ }^{\circ}$ Sig. 029. Idem.

N. ${ }^{\circ}$ Sig. 030. Idem.

Dim. Máx.: $2,3 \times 2,4 ; 1,5 \mathrm{H}$. cm.

(Borde de sección redondeada)

Dim. Máx.: $3 \times 2,9 ; 1,2 \mathrm{H} . \mathrm{cm}$.

(Borde de sección redondeada)

N. ${ }^{\circ}$ Sig. 031. Idem.

Dim. Máx.: $13,5 \times 4,5 ; 3,4 \mathrm{H} ; 15,5 \varnothing$ Máx. cm.

(Borde de sección redondeada)

N. ${ }^{\circ}$ Sig. 032. Fragmento borde de plato de sección redondeada. Presenta al interior filete al borde. Todo ello aplicado sobre esmalte blanco muy uniforme y compacto.

Dim. Máx:: 4,5×3,5; 2 H; 24,5 Ø Máx. cm.

N. ${ }^{\circ}$ Sig. 033. Idem.

Dim. Máx: $8 \times 5,5 ; 2,7 \mathrm{H} ; 22,8 \varnothing$ Máx. cm.

N. ${ }^{\circ}$ Sig. 034. Fragmento borde de plato de sección redondeada. Presenta al interior motivo vegetal. Todo ello aplicado sobre esmalte blanco.

Dim. Máx: $7,5 \times 4 ; 2 \mathrm{H} ; 16 \varnothing$ Máx. cm.

N. ${ }^{\circ}$ Sig. 035. Idem.

Dim. Máx: $12,5 \times 4 ; 2,5 \mathrm{H} ; 16 \varnothing$ Máx. cm. 
N. ${ }^{\circ}$ Sig. 036. Fragmento borde de plato de sección apuntada. Presenta al interior motivo decorativo indeterminado en azul y ocre. Todo ello aplicado sobre esmalte blanco.

Dim. Máx.: 3,5×1,7; 1,2 H; $18 \varnothing$ Máx. cm.

N. ${ }^{\circ}$ Sig. 037. Fragmento borde de plato de sección apuntada. Presenta al interior motivo vegetal. Todo ello aplicado sobre esmalte blanco.

Dim. Máx.: 4,5×3; $2 \mathrm{H} ; 19 \varnothing$ Máx. cm.

N. ${ }^{\circ}$ Sig. 038 . Idem.

Dim. Máx.: 4 ×5,2; $2 \mathrm{H} ; 15 \varnothing$ Máx. cm.

N. ${ }^{\circ}$ Sig. 039. Idem

Dim. Máx.: $8,7 \times 3 ; 1,6 \mathrm{H} ; 16,4 \varnothing$ Máx. cm.

(Borde de sección redondeada y esmalte irregular)

N. ${ }^{\circ}$ Sig. 040. Idem

Dim. Máx.: $4 \times 3,7 ; 1,5 \mathrm{H} ; 18 \varnothing$ Máx. cm.

N. ${ }^{\circ}$ Sig. 041. Idem

Dim. Máx.: $4,1 \times 3,5 ; 1,7$ H. cm

N. ${ }^{\circ}$ Sig. 042. Idem.

Dim. Máx.: $6,5 \times 4,3 ; 2,5 \mathrm{H} ; 18 \varnothing$ Máx. cm.

N. ${ }^{\circ}$ Sig. 043. Idem.

N. ${ }^{\circ}$ Sig. 044. Idem.

Dim. Máx.: $6,4 \times 4,5 ; 2,5$ H; $18 \varnothing$ Máx. cm.

Dim. Máx.: 4,5×5,2; 3,5 H; 18,4 Ø Máx. cm.

(Borde de sección redondeada, presentando también una incipiente base)

N. ${ }^{\circ}$ Sig. 045. Idem.

Dim. Máx.: $3,6 \times 4,3 ; 2,3 \mathrm{H}$. cm.

(Borde de sección redondeada, presentando también restos irregulares en azul al exterior)

N. ${ }^{\circ}$ Sig. 046. Fragmento de borde de sección redondeada. No presenta decoración alguna y consta de esmalte blanco muy claro, dejando entrever la pasta.

Dim. Máx.: $3 \times 4 ; 14, \varnothing$ Máx. cm.

N. ${ }^{\circ}$ Sig. 047. Idem.

Dim. Máx: $3 \times 3 \mathrm{~cm}$.

N. ${ }^{\circ}$ Sig. 048. Fragmentos correspondientes a un único plato de base plana y sección de borde redondeada. Presenta al interior decoración vegetal con cierto recargamiento hacia el borde. Todo ello aplicado sobre esmalte blanco.

Dim.: $19 \varnothing$ Máx; 9,4 $\varnothing$ Mín; 4 H. cm.

(Está conservado en un 40\%)

N. ${ }^{\circ}$ Sig. 049. Fragmento indeterminado. Presenta al interior motivo en ondas que forman una banda, remarcada por dos líneas, y otra banda formada por semicírculos unidos. Todo ello aplicado sobre esmalte blanco.

Dim. Máx.: $13,5 \times 12 \mathrm{~cm}$.

N. ${ }^{\circ}$ Sig. 050. Fragmento indeterminado. No presenta decoración. Esmaltado en blanco.

Dim. Máx: $4,5 \times 2 \mathrm{~cm}$.

N. ${ }^{\circ}$ Sig. 051. Fragmento indeterminado. Presenta al interior restos de decoración. Esmaltado en blanco con carácter irregular.

Dim. Máx.: $3,5 \times 3 \mathrm{~cm}$.

N. ${ }^{\circ}$ Sig. 052. Fragmento indeterminado. No presenta decoración. Esmaltado en blanco.

Dim. Máx.: $4 \times 3,5 \mathrm{~cm}$.

N. ${ }^{\circ}$ Sig. 053. Fragmento indeterminado. Presenta al interior decoración indeterminada en azul y amarillo. Todo ello aplicado sobre esmalte blanco.

Dim. Máx.: 2,8 $82,5 \mathrm{~cm}$. 

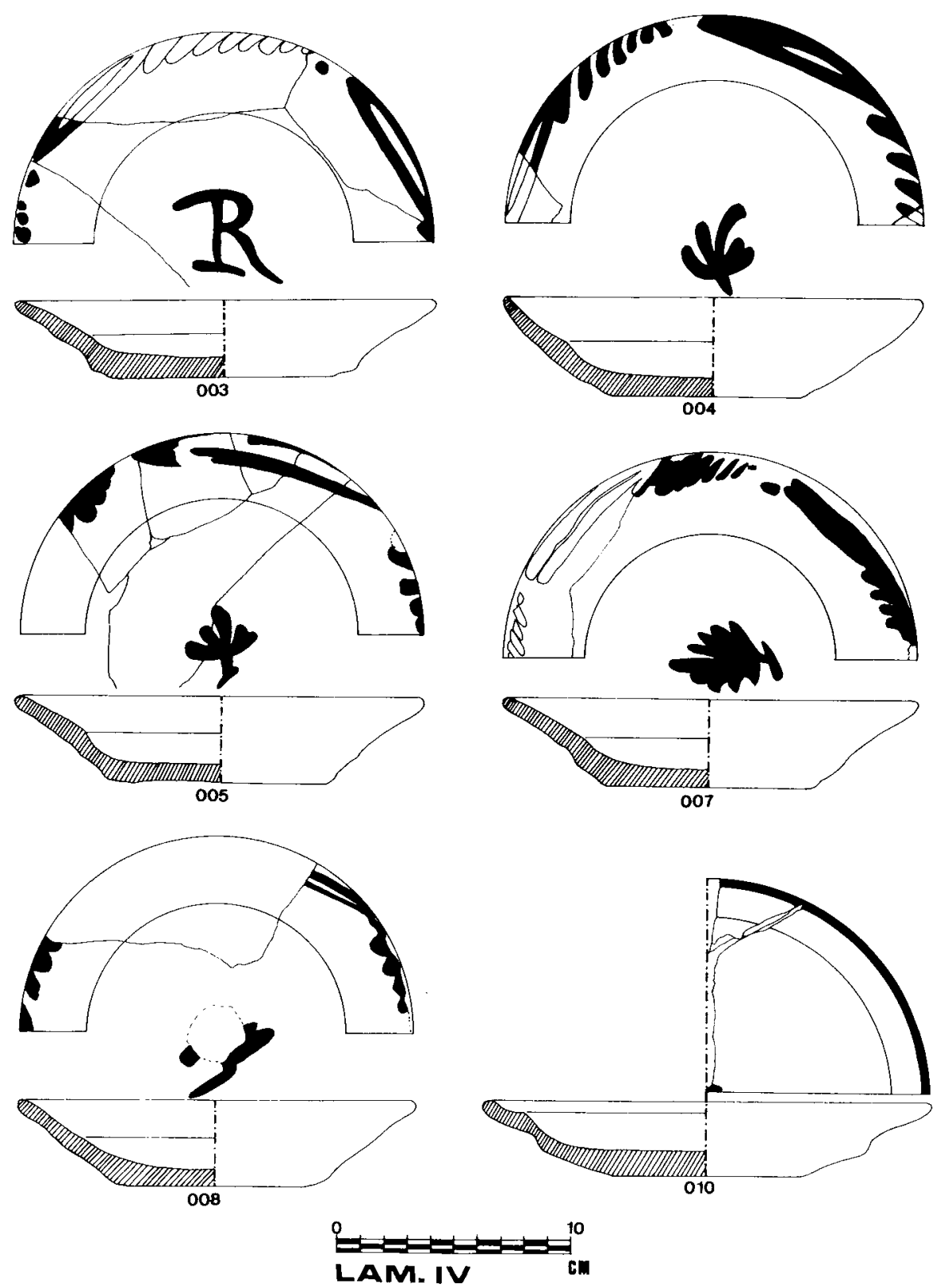

Lámina IV. Loza azul 

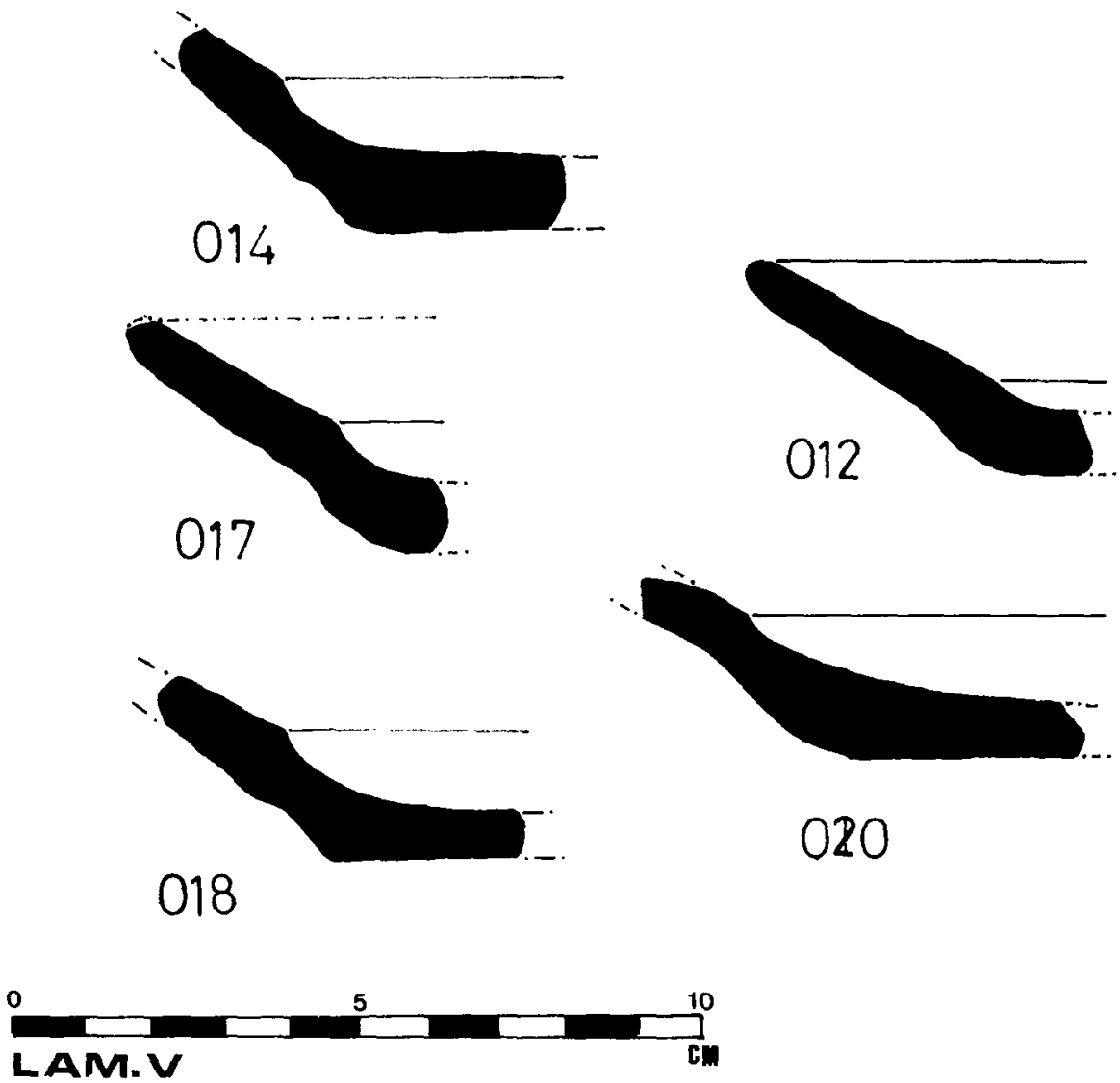

Lámina $\vee$. Secciones de bases de loza azul 


\section{NOTAS}

(1) Diario Información de 6 de Septiembre de 1983.

(2) IBARRA I RUIZ, P. «Convento de San José» Levante, n. ${ }^{\circ} 13$, año II, Elche, 10 de abril de 1926.

(3) IBARRA I RUIZ, P. Op. cit.

(4) RAMOS FOLQUÉS, A. Historia de Elche t. II, Elche 1971, p. 491.

(5) IBARRA I RUIZ, P. Op. cit.

(6) IBARRA I RUIZ, P. Op. cit.

(7) IBARRA I RUIZ, P. Op. cit.

(8) IBARRA I RUIZ, P. Op. cit.

(9) Archivo Histórico Municipal de Elche, Libro de Sitiadas 14, 18 de noviembre de 1650.

(10) IBARRA I RUIZ, P. Op. cit.

(11) SERRANO I JAÉN, J, «El temps dels senyors: la vila d'Elx entre dues cojuntures historiques (1262-1855): algunes notes». La rella $n .^{\circ} 3$. Elx 1984, pp. 23-49.

(12) A. H. M. E., Libro de Cabildos 88,31 de mayo de 1758.

(13) RAMOS FOLQUÉS, A. Op. cit., p. 492.

(14) A. H. M. E., Libro de Cabildos 157, 4 de enero de 1841. "Suplicando (a su Majestad) digne conceder a esta villa el referido edificio y su iglesia con el objeto de trasladar dicho Hospital de Caridad y establecer en el mismo Casa de Beneficencia».

(15) A. H. M. E., Cabildo de 12 de enero de 1438. «Mestre de fer teula e Rajola es vengut a la dita Vila dient q. si lo dit Consell li fa forn d'coure teula e Rajola franchament q. ell e muller e fills seus vendran per star en la dita e obrara del dit ofici. Per tal com la venguda del dit hom sia honorosa e profitosa a la dita vila. Hordena en tench per be que per lo obrer dels murs de la dita vila sia fet lo dit forn ab homens logats en aquell loch hon lo dit maestre designara e semylara aportant ans e primerament lo dit maestre llur muller e fills a la dita vila eço que lo dit obrer despendra en fer lo dit forn mostrant cautela li sia pres en compte lo qual dit obrer aja per llur salari per cascum dia dos sous".

RAMOS FOLQUES, A.: La industria, el comercio y la agricultura en Elche, Elche 1973, pág. 109.

IBARRA I RUIZ, P. Elche, materiales para su historia. Ensayos demostrativo de su antigüedad e importancia histórica. Cuenca 1926, pp. 145-148.

(16) A. H. M. E., Cabildo de 25 de abril de 1462. El Concejo ordena hacer un horno en la Plaça de Sant Jordi, trayendo un buen maestro moro que está en Elda y que quiere venir y dice que hay buena bierra «pera fer aci olles e canters llibrells e tases e llibrells e teules e Ralojes e altres coses d'obra d'terra".

(17) A. H. M. E., Cabildo de 20 de agosto de 1452. El Concejo ordena construir un horno de vidrio y casas para Nadal Torres, vidriero, en la Plaça de Sant Jordi. El puebio de la villa 
se queja de que el horno quedaría lejos y que estaría mejor que se hiciera cerca de donde estaba el antiguo horno. El Concejo así lo ordena.

(18) DE EPALZA, M. «Un modelo operativo de urbanismo musulmán Sharq Al-Andalus, $n .^{\circ}$ 2, Anales de la Universidad de Alicante 1985, p. 137-149.

(19) RAMOS FOLQUÉS, A. La industria... p. 110

(20) Vide nota 16.

(21) GOZÁLVEZ PÉREZ, V: La ciudad de Elche, Universidad de Valencia 1976, p. 78.

(22) SERRANO । JAÉN, J. Op. cit., p. 30.

(23) A. H. M. E., Cabildo de 3 de noviembre de 1853. «Se dió cuenta de un memorial de Vicente Segui vecino de Alicante, solicitando autorización para establecer una fábrica de Alfarería en terreno que pertenece al Hospital de Caridad de esta Villa, inmediato al ex-convento de San José, en el pasaje donde se dirigen las aguas del pozo de dicho edificio cuyas vertientes se obliga a mantener limpias y corrientes, como asi mismo a pagar la cantidad que se convenga, siempre que se le autorice para extraer de la acequia de Marchena la poca agua que ha de invertir en la citada fábrica, por la retribución que corresponde. Enterado el Cabildo acordó se pase original esta solicitud a la Junta Directiva de las aguas de la expresada acequia para que manifieste cuanto se le ofrezca y parezca y verificado se oyga sobre ello a la Junta Municipal de Beneficiencia a fin de poder resolver con acierto este negocio».

(24) Vide notas 15, 16, 23.

A. H. M. E., Cabildo de 26 de septiembre de 1530. «Frances Sánchez vehi de la ciutat de Oriola besa les mans de vostre magnificiençies a les quals fa asaber com ell suplicant sía mestre e tinga ofici de canterer y de fer scudelles y plats y tota altra obra vidriada..."

Cabildo de 21 de diciembre de 1530.

Cabildo de 22 de abril de 1533. Avecindamiento de "Luis de Baxa, mestre de fer canters» por cinco años.

Cabildo de 13 de marzo de 1585. «ltem per quant los dits magnifiches Justicia y jurats an fet venir a la present vila un canterer pera que fasa canters, plats y escudelles peral servici de dita vila y lian promes de donar casa franca y mes cinquanta reais pera ajuda de fer forn e considerat ques molt util y profit pera tots los vehins de dita vila de que en aquella se fasen dites obres...”

Cabildo de 14 de junio de 1642. El Concejo compra una casa para Francisco Esteban para que venga a hacer cántaros a la villa.

(25) A. H. M. E., Cabildo de 30 de marzo de 1487 . Se ordena se paguen 15 sueldos a Antonio de Ortiz por razón de seis meses que estuvo en las casas de aquél un maestro de hacer ollas.

Cabildo de 15 de mayo de 1491. Treinta sueldos a Gaspar Vives que se los prestó a Pedro Colomines, maestro de hacer ollas, cuando aquél vino a la Villa a hacer ollas.

(26) A. H. M. E., Cabildo de 21 de diciembre de 1677. Carlos Ortiz vende a Juan Calvo por ciento cuarenta libras la cantarería, por hallarse ésta en mal estado, con la condición de que se utilice perpetuamente para dicha industria.

(27) Vide nota 23.

(28) RUIZ TORRES, P. Señores y propietarios. Cambios en el sur del País Valenciano (16501850), Valencia 1981.

(29) A. H. M. E., Cabildo de 21 de mayo de 1684.

(30) LLOBREGAT, E. e IVARS, J. F. Història de l'art al Pais Valencià, València 1986, vol. I. p. 275.

(31) SESEÑA, N. Barros y lozas de España, Madrid 1976.

(32) PINEDO, C. y VIZCAINO, E. La cerámica de Manises en la Historia. León 1979.

(33) PINEDO C. y. VIZCAINO, E. Op. cit. 469-478.

SOLER M. P. "Loza valenciana de los siglos XVII y XVIIl», Revista Faenza, n. ${ }^{\circ}$ 5-6, pp.

(34) PINEDO, C. y VIZCAINO E. Op.cit. 


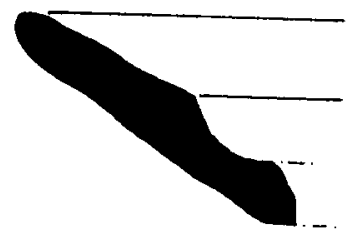

044

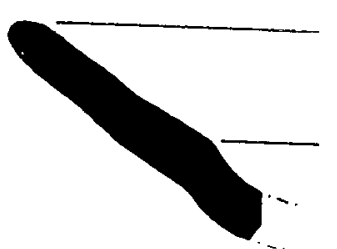

031
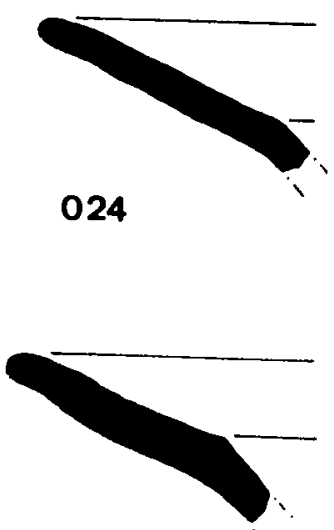

042

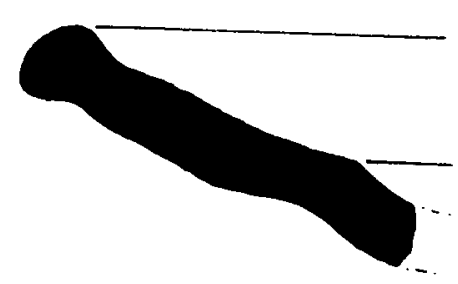

021

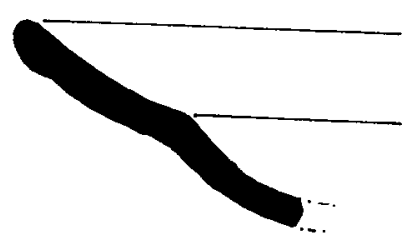

033

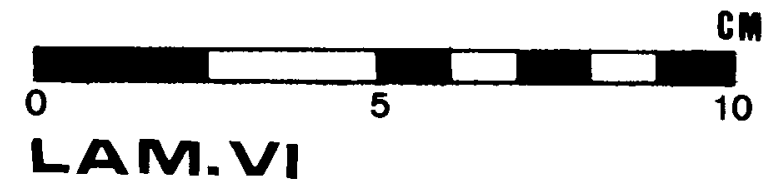

Lámina VI. Secciones de bordes de loza azul 